\title{
Effect of Lycopene on Doxorubicin-Induced Cardiotoxicity: An Echocardiographic, Histological and Morphometrical Assessment
}

\author{
Ana Lucia Anjos Ferreira ${ }^{1}$, Robert Mitchell Russell ${ }^{2}$, Noeme Rocha ${ }^{3}$, Marcelo Sady Placido Ladeira ${ }^{1}$, Daisy Maria Favero Salvadori ${ }^{4}$, \\ Maria Carolina Munhoz Oliveira Nascimento ${ }^{1}$, Mirna Matsui ${ }^{1}$, Flavio Augusto Carvalho ${ }^{1}$, Guangwen Tang ${ }^{2}$, \\ Luiz Shiguero Matsubara ${ }^{1}$ and Beatriz Bojikian Matsubara ${ }^{1}$
}

${ }^{1}$ Department of Internal Medicine, Botucatu Faculty of Medicine, Universidade Estadual Paulista, São Paulo State University, Botucatu, SP, Brazil, ${ }^{2}$ United States Department of Agriculture, Human Nutrition Research Center on Aging at Tufts University, Boston, MA, USA, ${ }^{3}$ Department of Pathology, Botucatu Faculty of Veterinary Medicine, Universidade Estadual Paulista, São Paulo State University, Botucatu, SP, Brazil and ${ }^{4}$ Department of Pathology, Botucatu Faculty of Medicine, Universidade Estadual Paulista, São Paulo State University, Botucatu, SP, Brazil

(Received November 23, 2006; Accepted February 2, 2007)

\begin{abstract}
Doxorubicin is an excellent chemotherapeutic agent utilized for several types of cancer but the irreversible doxorubicin-induced cardiac damage is the major limitation for its use. Oxidative stress seems to be associated with some phase of the toxicity mechanism process. To determine if lycopene protects against doxorubicin-induced cardiotoxicity, male Wistar rats were randomly assigned either to control, lycopene, doxorubicin or doxorubicin + lycopene groups. They received corn oil (control, doxorubicin) or lycopene $(5 \mathrm{mg} / \mathrm{kg}$ body weight a day) (lycopene, doxorubicin + lycopene) by gavage for a 7 -week period. They also received saline (control, lycopene) or doxorubicin $(4 \mathrm{mg} / \mathrm{kg})$ (doxorubicin, doxorubin + lycopene) intraperitoneally by week 3, 4, 5 and 6. Animals underwent echocardiogram and were killed for tissue analyses by week 7. Mean lycopene levels $(\mathrm{nmol} / \mathrm{kg})$ in liver were higher in the doxorubicin + lycopene group (5822.59) than in the lycopene group (2496.73), but no differences in lycopene were found in heart or plasma of these two groups. Lycopene did not prevent left ventricular systolic dysfunction induced by doxorubicin. However, morphologic examination revealed that doxorubicin-induced myocyte damage was significantly suppressed in rats treated with lycopene. Doxorubicin treatment was followed by increase of myocardium interstitial collagen volume fraction. Our results show that: (i) doxorubicin-induced cardiotoxicity was confirmed by echocardiogram and morphological evaluations; (ii) lycopene absorption was confirmed by its levels in heart, liver and plasma; (iii) lycopene supplementation provided myocyte protection without preventing interstitial collagen accumulation increase; (iv) doxorubicin-induced cardiac dysfunction was not prevented by lycopene supplementation; and (v) lycopene depletion was not observed in plasma and tissues from animals treated with doxorubicin.
\end{abstract}

Doxorubicin is used for the treatment of a wide variety of human tumours; however, the development of irreversible cardiotoxicity has limited its use [1]. Although doxorubicininduced cardiac injury appears to be multi-factorial, a common denominator is the cellular damage mediated by reactive oxygen species (ROS) [2]. Due to its success as a chemotherapeutic agent, several strategies including antioxidants have been tried to prevent or attenuate the cardiotoxicity of doxorubicin. Although it seems clear that ROS act in some phase of the doxorubicin-induced cardiotoxicity process, the studies using antioxidants have been showing conflicting results [3-9].

Lycopene is one of the most potent antioxidants among the dietary carotenoids due mainly to its many conjugated double bounds [10], and it also has the strongest singlet oxygenquenching ability compared to other carotenoids [11]. This singlet oxygen-quenching ability of lycopene is twice as high as that of $\beta$-carotene and 10 times higher than of $\alpha$-tocopherol

Author for correspondence: Ana Lucia Anjos Ferreira, Department of Clinical Medicine, Faculty of Medicine, Botucatu, Universidade Estadual Paulista, CEP 18618-970, Botucatu, SP, Brazil (fax +55 14 3882 2238, e-mail: ferreira@fmb.unesp.br).
[12]. Besides quenching singlet molecular oxygen and peroxyl radicals [13], strong interaction of lycopene has been shown to occur with other ROS such as $\mathrm{H}_{2} \mathrm{O}_{2}$ [14], which can generate the hydroxyl radical, known to induce membrane lipid peroxidation and DNA strand scission [15].

The relation between lycopene and cardiovascular disease has been examined in several epidemiological studies [16-19]. However, only two studies have evaluated the lycopene effect on doxorubicin-induced cardiotoxicity $[20,21]$ showing some protection. These were single dose anthracycline cardiotoxicity studies, which is quite a different condition from the multiple doses used in clinical situations.

The aim of this study was to evaluate the protective effect of lycopene supplementation on cardiotoxicity induced by multiple doses of doxorubicin. Additionally, we estimated lycopene uptake into tissues including the heart.

\section{Materials and Methods}

Chemical products. Doxorubicin hydrocloride was purchased from Pharmacia (Milan, Italy). All-trans- $\beta$-carotene (type IV), lycopene and ammonium acetate were purchased from Sigma Chemical Co. (St. Louis, MO, USA). Lutein was purchased from Kemin Industries 
(Des Moines, IA, USA). Zeaxanthin, cryptoxanthin, 13-cis- $\beta$-carotene, 9-cis- $\beta$-carotene and echinenone were kindly provided by HoffmannLa Roche (Nutley, NJ, USA). Lycopene from tomato oleoresin, Lyc-O-Mato, was a gift from LycoRed Natural Products Industries (Beer-Sheva, Israel). Solutions of carotenoids and retinoids were prepared under red light immediately before use. High pressure liquid chromatography (HPLC)-grade methanol and water were obtained from J. T. Baker Chemical (Philipsburg, NJ, USA), Methyltert-butyl ether was purchased from Aldrich Chemical (Milwaukee, WI, USA). All HPLC solvents were passed through a $0.45-\mathrm{mm}$ membrane filter and degassed before use. All carotenoid standards were stored at $-70^{\circ} \mathrm{C}$ until use. Pentobarbital sodium (Cristalia, Paulinia, SP, Brazil), quetamine cloridrate (Vetbrands, Jacarei, SP, Brazil) and xylazine (Bayer, Sao Paulo, SP, Brazil) were used for anaesthesia.

Animals. Male Wistar rats (370-400 g) from CEMIB (Centro Multidisciplinar de Investigação Biológica, UNICAMP, Campinas, SP, Brazil) were housed for 7 weeks and kept three per cage in plastic cages in the animal facility at the laboratories of Botucatu Faculty of Medicine, UNESP, where they consumed water and commercial diet described by Paiva et al. [22] ad libitum. Rats were randomly assigned either to the control group (control, $\mathrm{n}=24$ ), doxorubicin group $(n=40)$, lycopene group $(n=8)$ or doxorubicin + lycopene group $(\mathrm{n}=40)$. Intraperitoneal injections were done at week $3,4,5$ and 6; control and lycopene groups received an appropriate volume of $0.9 \% \mathrm{NaCl}$ intraperitoneally, while the doxorubicin and doxorubicin + lycopene received doxorubicin diluted in $0.9 \% \mathrm{NaCl}$ divided in four equal injections (each containing $4 \mathrm{mg} / \mathrm{kg}$ body weight). A cumulative dose of $16 \mathrm{mg} / \mathrm{kg}$ body weight was selected according to previous studies $[8,23]$. Lycopene or corn oil was daily given by gavage every morning for the entire 7 -week period. Lycopene oleoresin supplement mixed with corn oil $(5 \mathrm{mg}$ lycopene/kg body weight a day) was given to animals in the lycopene and doxorubicin + lycopene groups, while corn oil alone was given to the control and doxorubicin groups. The dose of lycopene used in this study was selected on the basis of previous studies [21,24]. To avoid differences in the energy provided, all groups received the same corn oil volume ( $\sim 0.5 \mathrm{ml} / \mathrm{kg}$ body weight a day). After the final intraperitoneal injections, all rats were observed for 1 week and then killed. This period was chosen based on the high mortality frequency observed after the final doxorubidin dose in a pilot study. One day before euthanasia all animals underwent a Transthoracic Doppler-echocardiogram using light anaesthesia (ketamine $50 \mathrm{mg} / \mathrm{kg}$ body weight) and xylazine (1 mg/kg body weight). Rats were fasted overnight before euthanasia under pentobarbital sodic $(50 \mathrm{mg} / \mathrm{kg}$ body weight intraperitoneally) anaesthesia and necropsied immediately after death. Tissues and plasma were collected and stored at $-80^{\circ} \mathrm{C}$ until carotenoid analyses. Tissues were fixed with $10 \%$ buffered formalin for $48 \mathrm{hr}$ for morphological analysis. A high number of animals were used in the control $(\mathrm{n}=40)$ and doxorubicintreated $(n=40)$ groups. These numbers were chosen because other organs from the control group would be utilized in another study and, also because of the elevated mortality $(>50 \%)$ in the doxorubidin groups that was observed in the pilot study. Twenty animals from the doxorubicin and 23 from the doxorubicin + lycopene groups were excluded from the study, because they died after a doxorubicin injection prior to protocol completion. This mortality rate is consistent with other reports $[8,25,26]$. The protocol used was in accordance with Ethical Principles for Animal Research adopted by Brazilian College of Animal Experimentation (COBEA) and was approved by the local Ethical Committee for Animal Research (protocol no. 267).

Lycopene preparation. Tomato oleoresin, Lyc-O-Mato 6\% dewaxed, was mixed with corn oil and stored at $4^{\circ}$ in the dark until used. It contained 13-cis- $\beta$-carotene (1\%), all-trans- $\beta$-carotene $(5 \%)$ and total lycopene $(95 \%)$. Of the total lycopene, $93 \%$ was in the all-trans form and $7 \%$ was as cis-lycopene isomers. The tomato oleoresin-corn oil mixture was stirred for $20 \mathrm{~min}$. in a water bath at $54^{\circ} \mathrm{C}$ before being fed to the animals. Each $\mathrm{ml}$ of solution contained $5 \mathrm{mg}$ total lycopene. Carotenoids were monitored at $450 \mathrm{~nm}$ and confirmed by diode-array spectra, as previously described [27]. The stability of lycopene in corn oil was checked by HPLC. Lycopene was stable in the tomato oleoresin corn oil mixture for 9 weeks at $-20^{\circ} \mathrm{C}$.

Carotenoid analyses of the diets. To each $10 \mathrm{~g}$ diet, $5 \mathrm{ml}$ of $\mathrm{H}_{2} \mathrm{O}$ were added and swirled for $1 \mathrm{~min}$. Extraction solution $(30 \mathrm{ml}$; hexane/acetone/ethanol/toluene 50:35:30:35) was added and swirled for $1 \mathrm{~min}$., followed by $16 \mathrm{hr}$ in the dark at room temperature; $4 \mathrm{ml}$ of $40 \%$ methanolic $\mathrm{KOH}$ and hexane $(30 \mathrm{ml})$ were added, and the mixture was left in the dark at room temperature. After $1 \mathrm{hr}, 10 \%$ $\mathrm{Na}_{2} \mathrm{SO}_{4}(30 \mathrm{ml})$ was added, shaken and left in the dark at room temperature. After 1 more hour, the hexane layer was collected and the volume was recorded. Aliquots of $200 \mu$ l were evaporated completely under $\mathrm{N}_{2}$, and the residue was redissolved in $100 \mu \mathrm{l}$ ethanol, vortexed and sonicated for $30 \mathrm{sec}$. A $50 \mu \mathrm{l}$ aliquot of the final extract was injected onto the HPLC system.

Plasma extraction. A $400 \mu \mathrm{l}$ aliquot of plasma was used for carotenoid analyses. $\mathrm{CHCl}_{3} / \mathrm{CH}_{3} \mathrm{OH}(3 \mathrm{ml} ; 2: 1, \mathrm{v} / \mathrm{v}), 500 \mu \mathrm{l}$ of $8.5 \mathrm{~g} / \mathrm{l}$ saline and $150 \mu \mathrm{l}$ of internal standard (retinyl acetate and echinonone) were added, and the mixture was vortexed and centrifuged for $10 \mathrm{~min}$. at $800 \times \mathrm{g}$ at $4^{\circ} \mathrm{C}$. Hexane $(3 \mathrm{ml})$ was added after collecting the lower layer. The chloroform and hexane layers were evaporated completely under $\mathrm{N}_{2}$, and the residue was redissolved in $150 \mu \mathrm{l}$ ethanol, vortexed and sonicated twice for $30 \mathrm{sec}$. A $50 \mu \mathrm{l}$ aliquot was used for HPLC analysis.

Tissue preparation and extraction. Heart and liver tissues were harvested, weighed and analysed after saponification as previously described [28]. Briefly, samples (150-200 g) were cut into small slices before the addition of $100 \mu \mathrm{l}(0.095 \mathrm{~mol} / \mathrm{l})$ pyrogallol in ethanol, $300 \mu \mathrm{l}(0.5 \mathrm{~mol} / \mathrm{l}) \mathrm{KOH}$ in $\mathrm{H}_{2} \mathrm{O}$ and $1 \mathrm{ml}$ ethanol. The mixture was vortexed and incubated at $37^{\circ} \mathrm{C}$ for $2 \mathrm{hr}$ while the tubes were covered with parafilm. After incubation, the samples were cooled to room temperature, $1 \mathrm{ml} \mathrm{H}_{2} \mathrm{O}$ was added and the mixture was revortexed. Echinenone in ethanol $(100 \mu \mathrm{l})$ was added as an internal standard. This mixture was shaken with $3 \mathrm{ml}$ anhydrous ether/hexane (2:1, stabilized with $1 \%$ ethanol v/v), then vortexed, and centrifuged at $800 \times \mathrm{g}$ at $4^{\circ} \mathrm{C}$ for $5 \mathrm{~min}$. The upper layer was removed, the extraction repeated and the upper layers were combined. $\mathrm{H}_{2} \mathrm{O}(2 \mathrm{ml})$ was added, the solution was vortexed and $2 \mathrm{ml}$ ethanol was added before centrifugation at $800 \times \mathrm{g}$ for $5 \mathrm{~min}$. The hexane layer was evaporated completely under $\mathrm{N}_{2}$, and the residue was redissolved in $100 \mu \mathrm{l}$ of ethanol, vortexed and sonicated twice for $30 \mathrm{sec}$. and centrifuged at $800 \times \mathrm{g}$ at $4^{\circ} \mathrm{C}$ for $2 \mathrm{~min}$. A $50 \mu \mathrm{l}$ aliquot was used for HPLC analysis. All sample analyses were done in duplicate. All sample processing was carried out under red light. The recovery of the added internal standard was consistently $>90 \%$.

HPLC analyses. The HPLC system was a Waters Alliance 2695 (Waters, Wilmington, MA, USA) and consisted of pump and chromatography bound to a 2996 programmable photodiode array detector and a 2475 fluorescence detector, a C30 carotenoid column (3 mm, $15034.6 \mathrm{~mm}$, YMC, Wilmington, MA, USA), and Empower software. The Waters 2996 programmable photodiode array detector was set at $340 \mathrm{~nm}$ for retinoids and $450 \mathrm{~nm}$ for carotenoids. The HPLC mobile phase was methanol/methyl-tert-butyl ether/water $(83: 15: 2, \mathrm{v} / \mathrm{v} / \mathrm{v}, 15 \mathrm{~g} / \mathrm{l}$ ammonium acetate in the water, solvent A) and methanol/methyl-tertbutyl ether/water (8:90:2, v/v/v, $10 \mathrm{~g} / \mathrm{l}$ ammonium acetate in the water, solvent $\mathrm{B})$. The gradient procedure, at a flow rate of $1 \mathrm{ml} / \mathrm{min}$. $\left(16^{\circ} \mathrm{C}\right.$ ), was as follows: (i) $100 \%$ solvent $\mathrm{A}$ was used for $2 \mathrm{~min}$. followed by a 6 -min. linear gradient to $70 \%$ solvent A; (ii) a 3 -min. hold followed by a 10 -min. linear gradient to $45 \%$ solvent A; (iii) a 2 -min. hold, then a 10 -min. linear gradient to $5 \%$ solvent A; (iv) a 4-min. hold, then a 2-min. linear gradient back to $100 \%$ solvent A. Using this method, lutein, zeaxanthin, cryptoxanthin, 
13-cis- $\beta$-carotene, all-trans- $\beta$-carotene, 9-cis- $\beta$-carotene, 2 -cis-lycopenes and all-trans-lycopene were adequately separated. Carotenoids and retinoids were quantified by determining peak areas in the HPLC chromatograms calibrated against known amounts of standards. The amounts were corrected for extraction and handling losses by monitoring the recovery of the internal standards.

Echocardiographic study. One week after final dose of doxorubicin therapy, all animals were evaluated in vivo by transthoracic echocardiography, using a SONOS 2000 (Hewlett-Packard Medical Systems, Andover, MA, USA) equipped with a $7.5-\mathrm{MHz}$ phased array transducer. Imaging was performed with a $60^{\circ} \mathrm{C}$ sector angle and $3 \mathrm{~cm}$ imaging depth. Rats were lightly anaesthetized by intramuscular injection with a mixture of ketamine $(50 \mathrm{mg} / \mathrm{kg})$ and xylazine $(1 \mathrm{mg} / \mathrm{kg})$. After shaving their chest, rats were placed in left decubitus position. Targeted two-dimensional M-mode echocardiograms were obtained from short-axis views of the left ventricle (LV) at or just below the tip of the mitral-valve leaflets, and at the level of aortic valve and left atrium. M-mode images of LV, left atrium (LA) and aorta were recorded on a black-and-white thermal printer (Sony Up-890MD, Cheshire, CT, USA) at a sweep speed of $100 \mathrm{~mm} / \mathrm{sec}$. All exams were performed by the same examiner and obtained according to the leading-edge method recommended by the American Society of Echocardiography [29]. Measurements represented the mean of at least five consecutive cardiac cycles. LV end-diastolic dimension (LVD) and posterior wall thickness were measured at maximal diastolic dimension, and the end-systolic dimension (LVS) was taken at maximal anterior motion of posterior wall. LA was measured at its maximal diameter. The peak velocities of diastolic transmitral flow (E and A velocities) were obtained in the apical four-chamber view and used as indexes of LV diastolic function. The time interval between two consecutive cardiac cycles was measured for heart rate calculation. Ventricular systolic function was evaluated by fractional shortening $(\mathrm{FS}, \%)=[(\mathrm{LVD}-\mathrm{LVS}) / \mathrm{LVD}] \times 100$.

Histological evaluation. Coronal sections of the left ventricle were fixed in $10 \%$ buffered formalin and embedded in paraffin. Fivemicrometre-thick sections were cut from the blocked tissue, and stained with haematoxylin and eosin and picrosirius red (Sirius red F3BA in aqueous saturated picric acid).

The severity of pathologic changes identified by haematoxylin and eosin was graded blindly by two pathologists on a scale from zero (no alterations) to three (marked abnormality) using semiquantitative analysis. The analyses considered the cellular appearance in necrosis, myocardial structure and infiltrative changes. The criteria used to identify cytoplasm necrosis were extreme acidophilic appearance, and myofibrillar loss, while nuclear necrosis was identified by pyknosis, karyorrhexis and karyolysis, corresponding to the size reduction, fragmentation and disappearance of nuclei, respectively. This investigations were performed using equipment from Carl Zeiss, Germany and consisted of a light microscope (Axio Imager A1, Zeiss, Oberkochen, Germany; magnification $\times 400$ ) attached to a digital video camera (Zeiss Vision, Oberkochen, Germany) and connected to a personal computer equipped with image analyser software (Axio Vision software rel., version 4.3).

The interstitial collagen volume fraction was determined using a whole slide of the stained heart with picrosirius red using an automated image analyser (Image-Pro Plus 3.0, Media Cybernetics, Silver Springs, MD, USA). The components of the cardiac tissue were identified according to their colour level: red for collagen fibres, yellow for myocytes and white for interstitial space. The digitized profiles were sent to a computer that calculated collagen volume fraction as the sum of all connective tissue areas divided by the sum of all connective tissue and myocyte areas. On average, 35 microscopic fields were studied with a X40 lens. Perivascular collagen was excluded from this analysis [30].

Statistical analysis. Results are expressed as mean \pm S.E. and the significance of differences were calculated by ANOva using SigmaStat version 2.0 for Windows (Jandel Scientific Software, San Rafael, CA, USA). For interstitial collagen volume fraction and transthoracic doppler-echocardiogram data two-way ANOVA was used to evaluate the effect of doxorubicin or lycopene and the interaction between the two treatments. A P level of 0.05 was used to determine significance.

\section{Results}

\section{Lycopene uptake and absorption.}

No lycopene was detected in the fed diets. From the tomato oleoresin corn oil mixture, the animals received 5.0, 0.77 and $2.0 \mathrm{mg} / \mathrm{kg}$ body weight a day) of total lycopene, $13-$ cis- $\beta$-carotene and all-trans- $\beta$-carotene, respectively. In the current study, lycopene was analysed as the total of both cis and trans isomers in tissues and plasma. There was no detectable lycopene in the plasma or tissues of the control or doxorubicin groups due to the lack of lycopene in fed diets. After 7 weeks of tomato oleoresin supplementation, the plasma levels of lycopene reached $4.69 \mathrm{nmol} / 1$ in the lycopene and $7.47 \mathrm{nmol} / 1$ in the doxorubicin + lycopene $(\mathrm{P}>0.05)$ groups. After tissue saponification, the lycopene level was higher in liver than in heart in both supplemented groups (lycopene and doxorubicin + lycopene). Liver lycopene concentrations were higher $(\mathrm{P}=0.009)$ in the doxorubicin + lycopene group $(5822.59 \mathrm{nmol} / \mathrm{kg})$ versus the lycopene group (2496.73 nmol/kg). However, the heart lycopene levels were not different between animals from the doxorubicin + lycopene $(48.59 \mathrm{nmol} / \mathrm{kg})$ and the lycopene $(44.99 \mathrm{nmol} / \mathrm{kg})$ groups (table 1).

\section{Effect of lycopene supplementation on general changes induced by doxorubicin.}

In addition to hair and weight loss and physical activity reduction due to doxorubicin therapy, most animals from the doxorubicin-treated groups at necropsy showed an evident cardiac softness and accumulation of serous fluid in pericardial pleural and peritoneal cavities. Animals from the control and lycopene groups showed no alterations in vivo or at necropsy. All groups had similar weight gain profiles up to week 4. After that, throughout the end of the protocol, the control and lycopene groups kept gaining weight, while

\section{Table 1.}

Concentrations of total lycopene in plasma and tissues in rats with and without tomato oleoresin supplementation at $5 \mathrm{mg}$ lycopene $/ \mathrm{kg}$ body weight/day/7 weeks) and doxorubicin therapy.

\begin{tabular}{lccc}
\hline Groups $^{\mathrm{n}}$ & Heart $^{\mathrm{a}} \mathrm{nmol} / \mathrm{kg}$ & Liver $^{\mathrm{a}} \mathrm{nmol} / \mathrm{kg}$ & Plasma nmol/1 \\
\hline $\mathrm{C}^{24}$ & $\mathrm{ND}$ & $\mathrm{ND}$ & $\mathrm{ND}$ \\
$\mathrm{L}^{8}$ & $44.99 \pm 5.08$ & $2496.73 \pm 720.769$ & $4.69 \pm 2.35$ \\
$\mathrm{D}^{20}$ & $\mathrm{ND}$ & $\mathrm{ND}$ & $\mathrm{ND}$ \\
$\mathrm{DL}^{17}$ & $48.59 \pm 4.61$ & $5822.59 \pm 779.59^{*}$ & $7.47 \pm 2.16$ \\
\hline
\end{tabular}

Values are means \pm S.E.; $n$, sample number; ND, not detectable; groups: L, lycopene; DL, doxorubicin + lycopene; Student's t-test was used to compare groups, ${ }^{*} \mathrm{P}=0.009$, significantly different from the liver of $\mathrm{L}$ group; ${ }^{\mathrm{a}} 0.095 \mathrm{~mol} / \mathrm{l}$ pyrogallol in ethanol and $0.5 \mathrm{~mol} / 1 \mathrm{KOH}$ in $\mathrm{H}_{2} \mathrm{O}$ for $2 \mathrm{hr}$ at $37^{\circ} \mathrm{C}$. 


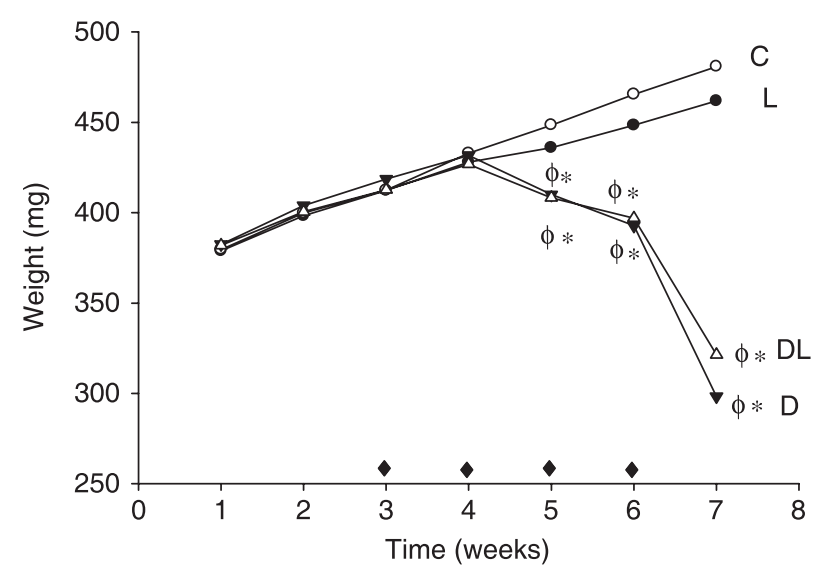

Fig. 1. Effect of lycopene supplementation and doxorubicin therapy on animal body weights. Values are means; groups: $\mathrm{C}$, control; L, lycopene; D, doxorubicin; DL, doxorubicin + lycopene; • doxorubicin $(4 \mathrm{mg} / \mathrm{kg}$ body weight intraperitoneally; cumulative dose $16 \mathrm{mg} / \mathrm{kg}$ body weight) or saline intraperitoneally; lycopene $(5 \mathrm{mg} / \mathrm{kg}$ body weight a day) or corn oil orally for 7 weeks; Student's t-test was used to compare groups: ${ }^{*}$ different from $\mathrm{C} ; \phi$ different from $\mathrm{L}$.

both doxorubicin-treated groups (doxorubicin and doxorubicin + lycopene) had a significant weight loss (control, lycopene versus doxorubicin, doxorubicin + lycopene; $\mathrm{P}<0.05$ ) (fig. 1). The animals from the control and lycopene groups did not die before the completion of the protocol; however, mortality was $50 \%$ and $58 \%$ in the doxorubicin and the doxorubicin + lycopene groups, respectively. Consumption of the lycopenecontaining corn oil mixture did not interfere with body weight evolution, mortality frequency or the necropsy findings of animals treated with or without doxorubicin.

\section{Effect of lycopene supplementation on doxorubicin-induced histological alterations.}

Rat myocardium from the control and lycopene groups showed no pathological changes. However, treatment with doxorubicin was followed by a multi-focal distribution of lesions when evaluated by haematoxylin and eosin. The most consistently and severely affected areas occurred in the myocardial tissue surrounding the major branches of the coronary vasculature. The lesions were characterized by (i) disorganization of myofibrillar morphology, vacuolization of myocytes, interstitial oedema, cytoplasmatic necrosis and mononuclear infiltrate and (ii) nuclear pyknosis, karyorrhexis and karyolysis. These same myocardial changes were identified in tissue from the animals treated with doxorubicin and supplemented with lycopene; however, they were reduced in number. When compared to the doxorubicin group, the blinded semi-quantitative analysis revealed significant less cytoplasmatic $(\mathrm{P}=0.002)$ and nuclear $(\mathrm{P}=$ $0.001)$ necrosis of the ventricular myocytes in the doxorubicin + lycopene animals. Although not significant, there was also a lower degree of internal arterial $(\mathrm{P}=0.054)$ and myocyte $(\mathrm{P}>0.05)$ hypertrophy in the doxorubicin + lycopene group as compared to the doxorubicin group. The main myocardial histologic alterations are shown in the fig. 2A-H. Myocardium assessed by picrosirius red staining showed that treatment with doxorubicin (the doxorubicin and the doxorubicn + lycopene groups) was accompanied by an increase of the interstitial collagen volume fraction when compared to animals treated otherwise $(\mathrm{P}<0.05)$. Lycopene supplementation had no effect on the collagen volume fraction, and there was no interaction between doxorubicin and lycopene treatments (fig. 3).

\section{Effect of lycopene supplementation on in vivo cardiac} alterations induced by doxorubicin.

Heart rate was similar among the groups (control 304 b.p.m.; lycopene 288 b.p.m.; doxorubicin 306 b.p.m., and doxorubicin + lycopene 307 b.p.m.). Echocardiographic exam allowed for evaluation of cardiac remodeling using morphological and functional variables (table 2). Doxorubicin treatment was associated to the significant decrease of end-diastolic LVD, diastolic left ventricle posterior wall thickness, left ventricle FS and transmitral flow early peak velocity (E). There was a significant interaction between doxorubicin and lycopene treatments in FS and end-systolic LVS. There was no lycopene influence on the identified changes found in the animals treated with doxorubicin.

\section{Discussion}

Doxorubicin is an antitumour drug that is useful in treating several types of cancer, although its clinical use has been restricted due to cardiomyopathy induced by dose-dependent cardiotoxicity. Once cardiomyopathy occurs, treatment options are few, and doxorubidin-induced heart failure is usually refractory to conventional therapy. Hence, prevention of doxorubicin-induced cardiomyopathy should be the goal. For that reason, we designed a protocol to evaluate the effect of lycopene supplementation on prevention of cardiotoxicity induced by doxorubicin.

In the current study, doxorubicin was injected for four times at $4 \mathrm{mg} / \mathrm{kg}$ resulting in a cumulative dose of $16 \mathrm{mg} / \mathrm{kg}$ equivalent to $500 \mathrm{mg} / \mathrm{m}^{2}$ of doxorubicin in a $50-\mathrm{kg}$ human being [31]. The current study design mimics the clinical situations where multiple doses of anthracycline are given to the patients. This kind of protocol used in this study usually yields a mortality ranging between $50-60 \%[8,25]$. In accordance, we registered a mortality of $50 \%$ and $58 \%$ in the doxorubicin and doxorubicin + lycopene groups, respectively. A high doxorubicin dose was used to support the hypotheses of lycopene protection; however, future studies will be required to better analyse the dose-effect of protection.

The dose of lycopene for supplementation was selected $(5 \mathrm{mg} / \mathrm{kg}$ a day) based on previous experimental reports that showed a protective effect of lycopene on doxorubicin cardiotoxicity $[20,21]$. Few studies have evaluated the effect of lycopene on doxorubicin-induced cardiotoxicity $[20,21]$. The investigators have shown that lycopene supplementation protects against heart lipid peroxidation, glutathione reduction [21] and the histological alterations $[20,21]$ induced by 

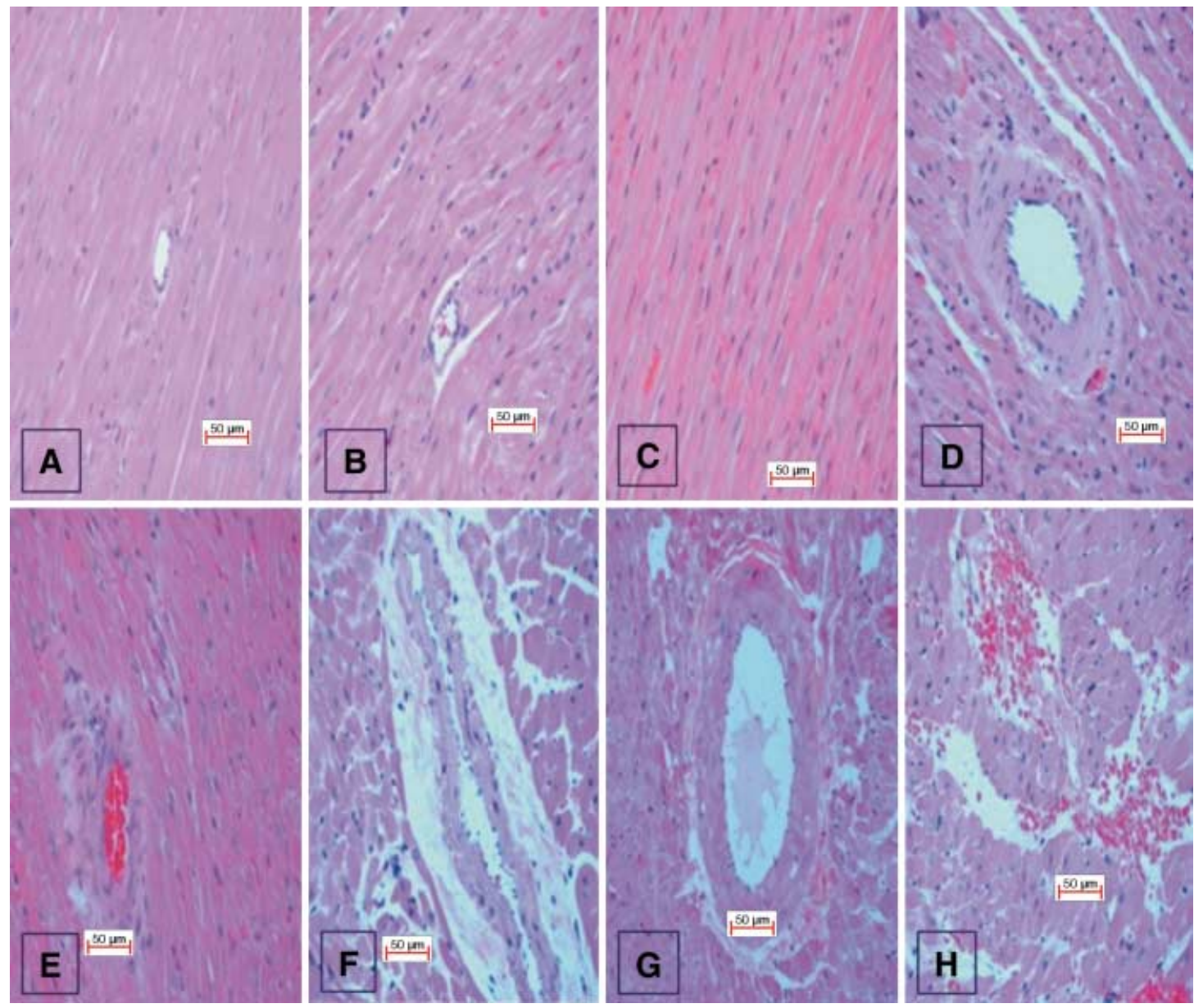

Fig. 2. Effect of lycopene supplementation (5 mg lycopene/kg body weight/day/7 weeks) and doxorubidin (cumulative dose $16 \mathrm{mg} / \mathrm{kg}$ body weight) histological alterations in rat myocardium. No histological abnormalities were found in control (A) and lycopene (B) groups. Treatment with doxorubicin was followed by extensive haemorrhage in the myocytic area $(\mathrm{H})$, hydropic degeneration $(\mathrm{G})$, necrosis $(\mathrm{F})$ and presence of mononuclear infiltration (E). This myocyte damage was significantly reduced in rats supplemented with lycopene (doxorubicin + lycopene group) (C and D). Paired t-tests showed differences between doxorubicin + lycopene and doxorubicin groups concerning cytoplasmatic $(\mathrm{F})(\mathrm{P}=0.002)$ and nuclear $(\mathrm{F})$ necrosis $(\mathrm{P}=0.001)$ (haematoxylin and eosin stain, original magnification $\times 400)$.

a single dose of doxorubicin, which is different from clinical conditions.

Most animals developed accumulation of serous fluid in the pericardial, pleural and peritoneal cavities. However, those findings were not interpreted as congestive failure, because the ventricular dysfunction detected by echocardiogram was not severe. Although we have not evaluated nephrotoxicity, it is relevant to suggest that such fluid accumulation may, in part, be due to hypoalbuminaemia, which is typically reported in patients [32] or rats [33] undergoing chemotherapy.

\section{Effect of lycopene supplementation on total lycopene levels in plasma and tissues.}

Oral treatment with $5.0 \mathrm{mg}$ lycopene $/ \mathrm{kg}$ body weight a day for 7 weeks resulted in the appearance of lycopene in plasma and in the saponified tissues (table 1). We detected lower plasma lycopene levels as compared to other studies in animals [28] or human beings [34]. Although few studies have evaluated cardiac lycopene, the levels found in the animals from the lycopene group are consistent with our pilot study that measured $42 \mathrm{nmol} / \mathrm{kg}$. A study using F344 rats found cardiac lycopene levels of $80 \mathrm{nmol} / \mathrm{kg}$ after 10 p.p.m. lycopene oleoresin had been incorporated into the diet for 8 weeks [35]. Another report using adult human heart obtained from autopsies showed higher heart lycopene levels. However, the history of lycopene supplementation to those patients is unknown [36]. As in other studies [36,37], the highest lycopene level was found in the liver. The higher number of low-density lipoprotein (LDL) receptors in the liver [38] than in heart [39] can explain the difference of lycopene levels between those organs. The lycopene liver concentration identified in present study is lower than in F344 rats and higher than ferrets supplemented with lycopene $(4 \mathrm{mg} / \mathrm{kg}$ body weight/9 weeks) [28]. The different results between our study and others above mentioned may be associated to the protocol used such as, lycopene cumulative doses, carotenoid extraction methods, strain and species of animals. 


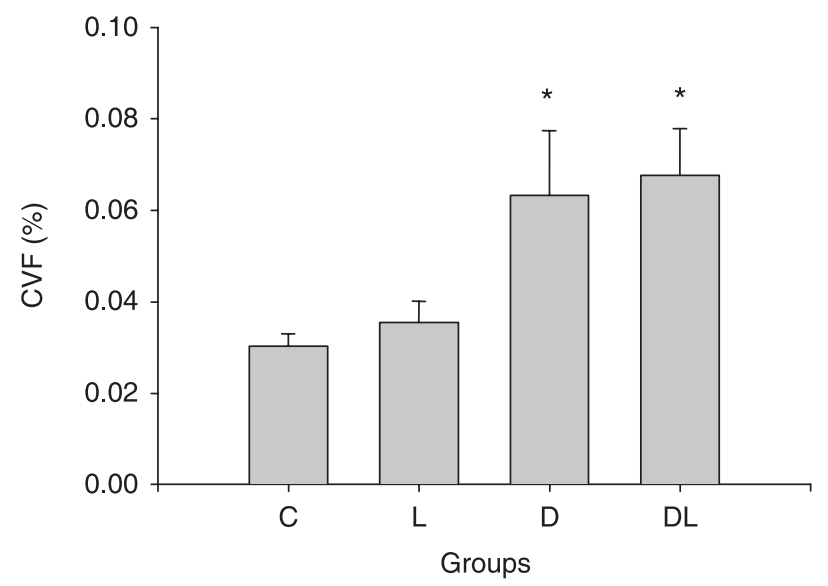

Fig. 3. Effect of lycopene supplementation and doxorubicin therapy on myocardium collagen volume fraction of rats. Values are means \pm S.E.; groups: $\mathrm{C}$, control; $\mathrm{L}$, lycopene; $\mathrm{D}$, doxorubicin; DL, doxorubicin + lycopene; doxorubicin $(4 \mathrm{mg} / \mathrm{kg}$ body weight intraperitoneally; cumulative dose $16 \mathrm{mg} / \mathrm{kg}$ body weight) or saline intraperitoneally; lycopene $(5 \mathrm{mg} / \mathrm{kg}$ body weight a day) or corn oil orally for 7 weeks; CVF, collagen volume fraction; Two-way ANOvA was used to analyse the treatment effects, ${ }^{*}=$ doxorubicin effect, $\mathrm{P}<0.05$.

\section{Effect of doxorubicin treatment on total lycopene levels in plasma and tissues.}

Doxorubicin therapy did not lower the lycopene level in tissues and plasma from lycopene-supplemented animals. Studies concerning doxorubicin-induced toxicity have reported both increased [40] and decreased lipid peroxidation after drug infusion [41]. The controversy about oxidative stress status $[42,43]$ is possibly due to the potential of two different physiopathological mechanisms, one for acute toxicity and another for chronic toxicity. The current results are in agreement with other studies where doxorubicin did not induce reduction of either lipophilic [8] or hydrophilic [43,44] antioxidant components in cardiac tissues. In fact, $\alpha$-tocoferol, retinol concentrations [8], catalase and superoxide dismutase activities [44] were all found to be unchanged in hearts from animals undergoing doxorubicin therapy in a similar experimental design (fractioned dose of $15 \mathrm{mg} / \mathrm{kg}$ doxorubicin). In addition, a number of studies using antioxidant supplement have failed to show protective effect on chronic cardiomyopathy induced by doxorubidin [3-5]. Inversely, acute protocols (single dose of doxorubicin) have yield a decrease of hydrophilic [21,42] and lipophilic [42] antioxidant components in cardiac tissues. These studies suggest that acute doxorubicin toxicity is related to oxidative stress, whereas the chronic doxorubicin administration is associated with other mechanisms of toxicity, such as decrease in energy production in the myocardium.

Thus, it has been recently suggested that chronic doxorubicin therapy may exert at least a part of its cardiotoxicity by delaying the long-chain fatty acid $\beta$-oxidation in heart [25], which is accompanied by increase of total cholesterol, triglycerides and LDL cholesterol in rat serum [45]. Because long-chain fatty acids are the major substrates for energy production in the aerobic adult myocardium [46], the inhibition of their $\beta$-oxidation could lead to cardiomyopathy due to energy supply deficiency [47].

It is well known that lycopene is transported more via LDL $(75 \%)$ than via high-density lipoprotein or very low-density lipoprotein [48]. Because doxorubicin can prevent fatty acid $\beta$-oxidation, we speculate that there would be more LDL available to transport lycopene, thereby leading to increased lycopene levels in serum and tissues exposed to doxorubicin action, even in organs with low LDL receptor numbers like the heart [39]. This assumption is supported by another study that reported higher liver $\alpha$-tocopherol levels in rat treated with doxorubicin when compared to control animals [8]. $\alpha$-Tocopherol is distributed among all lipoproteins, with a large proportion on the LDL fraction [49], which is what happens with lycopene as well.

Besides plasma and cardiac tissue, we also demonstrated that hepatic lycopene concentrations were not depleted in lycopene-supplemented animals after a fractioned dose of doxorubicin. Inversely, liver from animals receiving doxorubicin

Table 2.

Echocardiographic data after doxorubicin therapy in lycopene-supplemented rats.

\begin{tabular}{|c|c|c|c|c|c|c|c|}
\hline \multirow[b]{2}{*}{ Variables } & \multicolumn{4}{|c|}{ Groups $^{\mathrm{n}}$} & \multicolumn{3}{|c|}{ ANOva P-value } \\
\hline & $\mathrm{C}^{24}$ & $\mathrm{~L}^{8}$ & $\mathrm{D}^{20}$ & $\mathrm{DL}^{17}$ & $\begin{array}{l}\text { DOX } \\
\text { effect }\end{array}$ & $\begin{array}{l}\text { Lycopene } \\
\text { effect }\end{array}$ & $\begin{array}{c}\text { Interaction } \\
\text { effect }\end{array}$ \\
\hline $\mathrm{LA} \mathrm{mm}$ & $3.99 \pm 0.13$ & $4.47 \pm 0.33$ & $3.74 \pm 0.15$ & $4.02 \pm 0.19$ & 0.094 & 0.068 & 0.494 \\
\hline LVD mm & $7.58 \pm 0.14$ & $7.34 \pm 0.17$ & $6.45 \pm 0.17$ & $6.93 \pm 0.22$ & $<0.001$ & 0.548 & 0.073 \\
\hline LVS mm & $3.68 \pm 0.10$ & $3.10 \pm 0.13$ & $3.28 \pm 0.20$ & $3.72 \pm 0.29$ & 0.616 & 0.733 & 0.021 \\
\hline PWT mm & $1.41 \pm 0.03$ & $1.46 \pm 0.07$ & $1.07 \pm 0.05$ & $1.21 \pm 0.08$ & $<0.001$ & 0.148 & 0.458 \\
\hline FS \% & $0.51 \pm 0.01$ & $0.58 \pm 0.01$ & $0.50 \pm 0.02$ & $0.47 \pm 0.03$ & 0.007 & 0.368 & 0.049 \\
\hline $\mathrm{E} \mathrm{cm} / \mathrm{sec}$ & $68.57 \pm 1.62$ & $68.08 \pm 3.55$ & $59.64 \pm 2.95$ & $63.65 \pm 3.33$ & 0.029 & 0.559 & 0.456 \\
\hline $\mathrm{A} \mathrm{cm} / \mathrm{sec}$ & $44.22 \pm 1.65$ & $45.83 \pm 1.93$ & $44.23 \pm 3.67$ & $45.76 \pm 3.76$ & 0.991 & 0.62 & 0.989 \\
\hline
\end{tabular}

Vaues are means \pm S.E.; n, sample number; groups: C, control; L, lycopene; D, doxorubicin; DL, doxorubicin + lycopene; lycopene dose, $5 \mathrm{mg}$ lycopene/ $\mathrm{kg}$ body weight/day/7 weeks; doxorubicin cumulative dose, $16 \mathrm{mg}$ doxorubidin $/ \mathrm{kg}$ body weight; Two-way ANOvA was used to analyse effect of doxorubicin (DOX), lycopene and of the interaction between DOX and lycopene treatments; LA, left atrium diameter during ventricular systole; LVD, end-diastolic left ventricular diameter; LVS, end-systolic left ventricular diameter; PWT, diastolic left ventricle posterior wall thickness; FS, left ventricle fractional shortening; E, transmitral flow early peak velocity and A, transmitral flow late peak velocity. 
+ lycopene reached $42 \%$ higher lycopene levels than those without doxorubicin (the lycopene group) $(\mathrm{P}=0.009)$ indicating that the antioxidant status maintenance may be amplified by the high number of LDL receptors in hepatic tissue.

\section{Effect of lycopene supplementation on left ventricle histological changes induced by doxorubicin.}

The unequivocal histopathologic (haematoxylin and eosin) evidence of myocardial damage identified in doxorubicin group is in agreement with previous studies using animals [50,51] and patients [52] exposed to high doses of doxorubicin.

The present study showed that lycopene supplementation ( $5 \mathrm{mg} / \mathrm{kg} /$ day $/ 7$ weeks) (the doxorubicin + lycopene group) reduced the cardiac cellular changes induced by doxorubicin, indicating that lycopene contributes to the protection against myocyte-morphological injury, even though other substances present in tomato oleoresin $(\gamma$-tocopherol, $\alpha$-tocopherol, $\beta$-carotene, phytofluene and phytoene) [53] may also contribute to the observed changes.

Other studies using a doxorubicin acute toxicity model have shown a marked histological protective role of pretreatment with antioxidant supplement on cardiotoxicity [50,51], including lycopene [20,21]. The success of antioxidant treatment, apparently more evident in the acute than in the chronic model, indicates again that doxorubicin chronic cardiotoxicity pathogenesis may be accompanied by other events rather than ROS production.

The myocyte histological changes induced by doxorubicin (doxorubicin and doxorubicin + lycopene) were accompanied by a significant interstitial collagen volume fraction increase, which is in accordance with previous reports [51,54], although this is still matter of discussion [55]. Interestingly, we were able to show necrosis myocyte protection with no corresponding decrease in the interstitial collagen volume fraction. This finding suggests an abnormal collagen accumulation induced by other mechanism rather than a reparative process following necrosis such as reactive fibrosis as described previously in experimental hypertension $[56,57]$.

\section{Effect of doxorubicin treatment on haemodynamics and left ventricular remodeling.}

Left ventricle FS was significantly reduced in animals treated with doxorubicin, confirming systolic function impairment. The finding is in agreement with previous studies [58-61] with significant deterioration of FS after 4 weeks of doxorubicin treatment [62]. In the present study, there was no evidence of lycopene effect on FS until 1 week of doxorubicin therapy was completed. As far as we know, this is the first time that the role of lycopene is assessed by echocardiography in doxorubicin-induced cardiotoxicity. In human beings, diastolic dysfunction seems to precede systolic dysfunction in doxorubicin-induced cardiotoxicity [63,64]. Conversely, our findings suggest systolic rather than diastolic function impairment. It has been pointed out that reduction of early diastolic transmitral flow velocity would be interpreted as an initial sign of relaxation impairment. The lack of experi- mental studies analysing this issue in doxorubicin-induced cardiotoxicity prevents further comparisons. The PWT decrease identified in animals treated with doxorubicin is in agreement with human studies [65,66], reflecting chronic cardiotoxicity. The significant interaction between doxorubicin and lycopene treatments on FS is interesting, as it indicates a deleterious synergism in left ventricle function.

Histological and functional data suggest that lycopene protection against cellular-morphological damage was not sufficient to prevent ventricular dysfunction and mortality in the current experimental protocol. Therefore, it seems reasonable to assume that doxorubicin causes other alterations besides cellular necrosis. In addition, the maintenance of lycopene levels in plasma and myocardium from lycopenesupplemented rats treated with doxorubicin suggest that the long-term doxorubicin therapy is more related to other mechanisms than lycopene consumption, such as decrease in energy production, which was not examined in the present study.

Further studies will be required to clarify the mechanism beneath the preserved lycopene levels in heart, liver and plasma after doxorubicin treatment. The assumption that antioxidant defences can be maintained in this situation should encourage studies on new therapeutic strategies to limit the toxicity of this and other important antitumour drugs. We must admit that although the experimental design using multiple doses of doxorubicin did mimic the clinical situation, it would be imprudent to extrapolate the current results to human beings, and future studies are necessary to test the doxorubicin antitumour action under lycopene supplementation.

\section{Acknowledgements}

We thank Fundação de Amparo à Pesquisa do Estado de São Paulo, São Paulo, SP, Brazil, for financial support (FAPESP 02-9800-05). We also thank Alexandre L. Loureiro, Anita Modenese, Camila Correa, Elenize J. Pereira, José A. Souza, José C. Georgete, Jian Qin, Mário A. Dallaqua, Mário B. Bruno, Rogério A. Monteiro, Sandra A. Fábio, Sueli Clara, Vítor M. Souza for technical assistance and LycoRed Natural Products Industries, Beer-Sheva, Israel for supplying the tomato oleoresin.

\section{References}

1 Buja LM, Ferrans VJ, Mayer RJ, Roberts WC, Henderson ES. Cardiac ultraestructural changes induced by daunorubicin therapy. Cancer 1973;32:771-88.

2 Li T, Danelisen I, Singal PK. Early changes in myocardial antioxidant enzymes in rats treated with adriamycin. Mol Cell Biochem 2002;232:19-26.

3 Van Vleet JF, Ferrans VJ, Weirich WE. Cardiac disease induced by chronic adriamycin administration in dogs and an evaluation of vitamin E and selenium as cardioprotectants. Am J Pathol 1980;99:13-42.

4 Breed JG, Zimmerman AN, Dormans JA, Pinedo HM. Failure of the antioxidant vitamin $\mathrm{E}$ to protect against adriamycininduced cardiotoxicity in the rabbit. Cancer Res 1980;40:20338 . 
5 Legha SS, Wang YM, Mackay B et al. Clinical and pharmacologic investigation of the effects of alpha-tocopherol on adriamycin cardiotoxicity. Ann N Y Acad Sci 1982;393:411-8.

6 Myers C, Bonow R, Palmeri S et al. A randomized controlled trial assessing the prevention of doxorubicin cardiomyopathy by N-acetylcysteine. Semin Oncol 1983;10:53-5.

7 Wahab MHA, Akoul EEMS, Abdelaziz AH. Modulatory effects of melatonin and vitamin-E on doxorubicin-induced cardiotoxicity in Ehrlich ascites carcinoma bearing mice. Tumori 2000;86:157-62.

8 Danelisen I, Palace V, Lou H, Singal PK. Maintenance of myocardial levels of vitamin A in heart failure due to adriamycin. J Mol Cell Cardiol 2002;34:789-95.

9 Wattanapitayakul SK, Chularojmontri L, Herunsalee A, Charuchongkolwongse S, Niumsakul S, Bauer JA. Screening of antioxidants from medicinal plants for cardioprotective effect against doxorubicin toxicity. Basic Clin Pharmacol Toxicol 2005;96:80-7.

10 Stahl W, Sies H. Physical quenching of singlet oxygen and cis-trans isomerization of carotenoids. Ann N Y Acad Sci 1993;691:10-9.

11 Di Mascio P, Kaiser S, Sies H. Lycopene as the most efficient biological carotenoid singlet oxygen quencher. Arch Biochem Biophys 1989;274:532-8.

12 Agarwal S, Rao VA. Tomato lycopene and its role in human health and chronic diseases. Can Med Assoc J 2000;163:739-44.

13 Stahl W, Sies H. Antioxidant activity of carotenoids. Mol Aspects Med 2003;24:345-51.

14 Wang S, Konorev EA, Kotamraju S, Joseph J, Kalivendi S, Kalyanaraman B. Doxorubicin induces apoptosis in normal and tumor cells via distinctly different mechanisms. Intermediacy of $\mathrm{H}_{2} \mathrm{O}_{2}$ - and p53-dependent pathways. J Biol Chem 2004;279:25535-43.

$15 \mathrm{Lu} \mathrm{Y}$, Etoh $\mathrm{H}$, Watanabe $\mathrm{N}$ et al. A new carotenoid, hydrogen peroxide oxidation products from lycopene. Biosci Biotech Biochem 1995;59:2153-5.

$16 \mathrm{Wu} \mathrm{K}$, Schwartz SJ, Platz EA et al. Variations in plasma lycopene and specific isomers over time in a cohort of U.S. men. J Nutr 2003;133:1930-6.

17 Kardinaal AF, Kok FJ, Ringstad J et al. Antioxidants in adipose tissue and risk of myocardial infarction: the EURAMIC Study. Lancet 1993;342:1379-84.

18 Kohlmeier L, Kark JD, Gomez-Gracia E et al. Lycopene and myocardial infarction risk in the EURAMIC Study. Am J Epidemiol 1997;146:618-26.

19 Kristenson M, Zieden B, Kucinskiene Z et al. Antioxidant state and mortality from coronary heart disease in Lithuanian and Swedish men: concomitant cross sectional study of men aged 50. Br Med J 1997;314:629-33.

20 Karimi G, Ramezani M, Abdi A. Protective effects of lycopene and tomato extract against doxorubicin-induced cardiotoxicity. Phytother Res 2005;19:912-4.

21 Yilmaz S, Atessahin A, Sahna E, Karahan I, Ozer S. Protective effect of lycopene on adriamycin-induced cardiotoxicity and nephrotoxicity. Toxicology 2006;218:164-71.

22 Paiva SA, Novo R, Matsubara BB et al. Beta-carotene attenuates the paradoxical effect of tobacco smoke on the mortality of rats after experimental myocardial infarction. J Nutr 2005;135:2109 13 .

23 Siveski-Iliskovic N, Hill M, Chow DA, Singal PK. Probucol protects against adriamycin cardiomyopathy without interfering with its antitumor effect. Circulation 1995:91:10-5.

24 Cohen LA. A review of animal model studies of tomato carotenoids, lycopene, and cancer chemoprevention. Exp Biol Med 2002;227:864-8.

25 Abdel-aleem S, el-Merzabani MM, Sayed-Ahmed M, Taylor DA, Lowe JE. Acute and chronic effects of adriamycin on fatty acid oxidation in isolated cardiac myocytes. J Mol Cell Cardiol 1997;29:789-97.

26 Matos HR, Capelozzi VL, Gomes OF, Di Mascio P, Medeiros MHG. Lycopene inhibits DNA damage and liver necrosis in rats treated with ferric nitrilotriacetate. Arch Biochem Biophys 2001;396:171-7.

27 Yeum KJ, Lee-Kim YC, Yoon S et al. Similar metabolites formed from $\beta$-carotene by either human gastric mucosal homogenates, lipoxygenase or linoleic acid hydroperoxide. Arch Biochem Biophys 1995;321:167-74

28 Ferreira ALA, Yeum KJ, Liu C et al. Tissue distribution of lycopene in ferrets and rats after lycopene supplementation. J Nutr 2000;130:1256-60.

29 Sahn DJ, DeMaria A, Kisslo J, Weyman AE. The Committee on M-mode standardization of the American Society of Echocardiography. Recommendations regarding quantitation in M-mode echocardiography: results of a survey of echocardiographic measurements. Circulation 1978;58:1072-83.

30 Matsubara LS, Matsubara BB, Okoshi MP, Cicogna AC, Janicki JS. Alterations in myocardial collagen content affect rat papillary muscle function. Am Physiol Heart Circ Physiol 2000;279:H1534-9.

31 Hiroe M, Ohta Y, Fujita N et al. Myocardial uptake of 111 In monoclonal antimyosin Fab in detecting doxorubicin cardiotoxicity in rats. Morphological and hemodynamic findings. Circulation 1992;86:1965-72.

32 Deepa PR, Varalakshmi P. The cytoprotective role of a lowmolecular-weight heparin fragment studied in an experimental model of glomerulotoxicity. Eur J Pharmacol 2003;478:199-205.

33 Cusack BJ, Muslin PS, Johnson CJ, Vestal RE, Olson RD. Aging increases the cardiotoxicity of daunorubicin and daunorubicinol in the rat. J Gerontol A Biol Sci Med Sci 1996;51:B376-84.

34 Stahl W, Sies H. Uptake of lycopene and its geometrical isomers is greater from heat-processed than from unprocessed tomato juice in humans. J Nutr 1992;122:2161-6.

35 Jain CK, Agarwal S, Rao AV. The effect of dietary lycopene on bioavailability, tissue distribution, in vivo antioxidant properties and colonic preneoplasia in rats. Nutr Res 1999;19:1383-91.

36 Kaplan LA, Lau JM, Stein EA. Carotenoid composition, concentrations, and relationships in various human organs. Clin Physiol Biochem 1990;8:1-10.

37 Narisawa T, Fukaura Y, Hasebe M et al. Prevention of Nmethylnitrosourea-induced colon carcinogenesis in F344 rats by lycopene and tomato juice rich in lycopene. Jpn J Cancer Res 1998;89:1003-8.

38 Clinton SK, Emenhiser C, Schwartz SJ et al. cis-trans lycopene isomers, carotenoids, and retinol in the human prostate. Cancer Epidemiol Biomarkers Prev 1996;5:823-33.

39 Osono Y, Woollett LA, Herz J, Dietschy JM. Role of the low density lipoprotein receptor in the flux of cholesterol through the plasma and across the tissues of the mouse. J Clin Invest 1995;95:1124-32.

40 Quiles JL, Ramirez-Tortosa MC, Huertas JR et al. Olive oil supplemented with vitamin $\mathrm{E}$ affects mitochondrial coenzyme $Q$ levels in liver of rats after an oxidative stress induced by adriamycin. Biofactors 1999;9:331-6.

41 Minotti G, Mancuso C, Frustaci A et al. Paradoxical inhibition of cardiac lipid peroxidation in cancer patients treated with doxorubicin. Pharmacologic and molecular reappraisal of anthracycline cardiotoxicity. J Clin Invest 1996;98:650-61.

42 Deepa PR, Varalakshmi P. Protective effect of low molecular weight heparin on oxidative injury and cellular abnormalities in adriamycin-induced cardiac and hepatic toxicity. Chem Biol Interact 2003;146:201-10.

43 Dziegiel P, Murawska-Cialowicz E, Jethon $\mathrm{Z}$ et al. Melatonin stimulates the activity of protective antioxidative enzymes in 
myocardial cells of rats in the course of doxorubicin intoxication. J Pineal Res 2003;35:183-7.

44 Li T, Danelisen I, Belló-Klein A, Singal PK. Effects of probucol on changes of antioxidant enzymes in adriamycin-induced cardiomyopathy in rats. Cardiovasc Res 2000;46:523-30.

45 Siveski-Iliskovic N, Singal PK. Lipid lowering: an important factor in preventing adriamycin-induced heart failure. Am J Pathol 1997;150:727-34.

46 Neely JR, Morgan HE. Relationship between carbohydrate and lipid metabolism and the energy balance of heart muscle. Annu Rev Physiol 1974;36:413-59.

47 Corr PB, Gross RS, Sobel BE. Amphipathic metabolites and membrane dysfunction in ischemic myocardium. Circ Res 1985;55:135-54.

48 Krinsky NI, Cornewell DG, Oncley JL. The transport of vitamin $\mathrm{A}$ and carotenoids in human plasma. Arch Biochem Biophys 1958;73:233-46.

49 Esterbauer H, Jurgens G, Quehenberger O, Koller E. Autoxidation of human low density lipoprotein: loss of polyunsaturated fatty acids and vitamin $\mathrm{E}$ and generation of aldehydes. J Lipid Res 1987;28:495-509.

50 Saad SY, Najjar TA, Al-Rikabi AC. The preventive role of deferoxamine against acute doxorubicin-induced cardiac, renal and hepatic toxicity in rats. Pharmacol Res 2001;43:211-8.

51 Yagmurca M, Fadillioglu E, Erdogan H, Ucar M, Sogut S, Irmak MK. Erdosteine prevents doxorubicin-induced cardiotoxicity in rats. Pharmacol Res 2003;48:377-82.

52 Billingham ME, Mason JW, Bristow MR, Daniels JR. Anthracycline cardiomyopathy monitored by morphologic changes. Cancer Treat Rep 1978;62:865-72.

53 Richelle M, Bortlik K, Liardet S et al. A food-based formulation provides lycopene with the same bioavailability to humans as that from tomato paste. J Nutr 2002;132:404-8.

54 Tokudome T, Mizushige K, Noma T et al. Prevention of doxorubicin (adriamycin)-induced cardiomyopathy by simultaneous administration of angiotensin-converting enzyme inhibitor assessed by acoustic densitometry. J Cardiovasc Pharmacol 2000;36:361-8.

55 Caulfield JB, Bittner V. Cardiac matrix alterations induced by adriamycin. Am J Pathol 1988;133:298-305.
56 Jalil JE, Doering CW, Janicki JS, Pick R, Shroff SG, Weber KT. Fibrillar collagen and myocardial stiffness in the intact hypertrophied rat left ventricle. Circ Res 1989;64:1041-50.

57 Brilla CG, Weber KT. Reactive and reparative myocardial fibrosis in arterial hypertension in the rat. Cardiovasc Res 1992;26:671-7.

58 Delgado RM, Nawar MA, Zewail AM et al. Cyclooxygenase-2 inhibitor treatment improves left ventricular function and mortality in a murine model of doxorubicin-induced heart failure. Circulation 2004;109:1428-33.

59 Ha JW, Kang SM, Pyun WB et al. Serial assessment of myocardial properties using cyclic variation of integrated backscatter in an adriamycin-induced cardiomyopathy rat model. Yonsei Med J 2005;46:73-7.

60 de Andrade JN, Camacho AA, Santos PS, Fantinatti AP, Nunes N, Stopiglia AJ. Plication of the free wall of the left ventricle in dogs with doxorubicin-induced cardiomyopathy. Am J Vet Res 2005;66:238-43.

61 Katona M, Boros K, Santha P, Ferdinandy P, Dux M, Jancso G. Selective sensory denervation by capsaicin aggravates adriamycin-induced cardiomyopathy in rats. Naunyn Schmiedebergs Arch Pharmacol 2004;370:436-43.

62 Koh E, Nakamura T, Takahashi H. Troponin-T and brain natriuretic peptide as predictors for adriamycin-induced cardiomyopathy in rats. Circ J 2004;68:163-7.

63 Sung RY, Huang GY, Shing MK et al. Echocardiographic evaluation of cardiac function in paediatric oncology patients treated with or without anthracycline. Int J Cardiol 1997;60:239-48.

64 Marchandise B, Schroeder E, Bosly A et al. Early detection of doxorubicin cardiotoxicity: interest of Doppler echocardiographic analysis of left ventricular filling dynamics. Am Heart J 1989;118:92-8.

65 Lipshultz SE, Lipsitz SR, Mone SM et al. Female sex and drug dose as risk factors for late cardiotoxic effects of doxorubicin therapy for childhood cancer. N Engl J Med 1995;332:1738-43.

66 Paiva MG, Petrilli AS, Moises VA, Macedo CR, Tanaka C, Campos O. Cardioprotective effect of dexrazoxane during treatment with doxorubicin: a study using low-dose dobutamine stress echocardiography. Pediatr Blood Cancer 2005;45:902-8. 